\title{
CFD/CSD COUPLING FOR AN ISOLATED ROTOR USING PRECICE
}

\section{Qunsheng Huang ${ }^{1}$, Amine Abdelmoula ${ }^{2}$, Gerasimos Chourdakis ${ }^{1}$, Juergen Rauleder ${ }^{3}$, Benjamin Uekermann ${ }^{4}$}

\author{
${ }^{1}$ Department of Informatics, Technical University of Munich \\ \{huangq, chourdak\}@in.tum.de \\ ${ }^{2}$ Institute of Helicopter Technology, Technical University of Munich \\ amine. abdelmoulaetum.de \\ ${ }^{3}$ Georgia Institute of Technology \\ juergen.rauleder@gatech.edu \\ ${ }^{4}$ Institute for Parallel and Distributed Systems, University of Stuttgart \\ benjamin. uekermann@ipvs.uni-stuttgart.de
}

Key words: Multi-physics, Fluid-Structure Interaction, Rotors, CFD/CSD Coupling

\begin{abstract}
Modeling a rotor blade flow field involves computing the blade motion, elastic deformation, and the three-dimensional forces and moments for specific trim conditions. Such a complex multiphysics problem, which includes a strong fluid-structure interaction, should be modeled by coupling separate solvers which are specialized on solving single-physics problems. In this work, we present a modular and extensible TAU-CAMRAD II coupling environment using the preCICE coupling library [1]. In this coupling, the aerodynamic forces and moments were computed with the CFD solver TAU. The blade control angle for the CFD simulation were determined by the CSD solver CAMRAD II. We validated the implementation using a modified model of the HART-II rotor at an advancing ratio of $\mu=0.3$. Besides the potential that this work unlocks for future simulations of an active rotor, it also serves as an example of using preCICE for geometric multi-scale (1D-3D) coupling of closed-source solvers for periodic phenomena.
\end{abstract}

\section{INTRODUCTION}

The simulation of a flow field passing by helicopter rotor blades is a challenging task that requires the consideration of the aerodynamic forces along with the motion and elastic deformation of the rotor blades. Since these structural and aerodynamic entities are strongly interlinked, one of the major difficulties in modeling such a complex multi-physics problem is to obtain an accurate approximation.

In this work, we coupled a computational structural dynamics (CSD) solver and a computational fluid dynamics (CFD) solver following a loose coupling approach. The forces and moments passed from the CFD to CSD are based on the the delta airloads method (see, for example, Pahlke et al., Servera et al. and Potsdam et al. [2, 3, 4]). This method was implemented within the scope of the loose coupling of CAMRAD II [5] and OVERFLOW, presented by Potsdam et al. [4] and the comprehensive direct com- 
parison of loosely- and tightly-coupled rotor blade, provided by Altmikus et al. [6]. More investigations using the delta airloads method continued through the mid 2000s with Abras et al. experimenting with the use of unstructured grids in loosely-coupled simulations of the UH-60A rotors [7]. A series of NASA funded wind tunnel tests were run in May 2010 and the results were used as validation data for a series of loosely-coupled simulations: Marpu et al. coupled a hybrid CFD solver, GT-Hybrid, with DYMORE [8] and Lee-Rausch et al. performed their investigations with a FUN3D-CAMRAD II coupling [9].

Recently, the Institute of Helicopter Technology at the Technical University of Munich (TUM) coupled TAU [10] and CAMRAD II [11]. However, this coupling was customized to a specific simulation case. Therefore, significant work is needed to extend it to take advantage of scalable communication methods, accurate mapping techniques, and efficient coupling schemes. In this work, our goal is to develop a general, easy-to-use, and modular coupling environment for helicopter simulations, making such simulations easier to set up, faster to solve, and easier to maintain. The aim of this work is to establish a basis for future, fully-resolved fluid-structure interaction simulations. In this context, we present a proof-of-concept for helicopter fluid-structure interaction simulations using preCICE ${ }^{1}$, a free/opensource, minimally invasive, black-box coupling library, which already implements advanced communication, mapping, and coupling algorithms. At the same time, preCICE has been used in a plethora of FSI studies outside the rotorcraft field, as demonstrated in the preCICE reference paper [1] and by the preCICE community ${ }^{2}$. With the various FSI examples from different fields at hand, the question of whether preCICE can also be used for rotorcraft FSI simulations becomes legitimate.

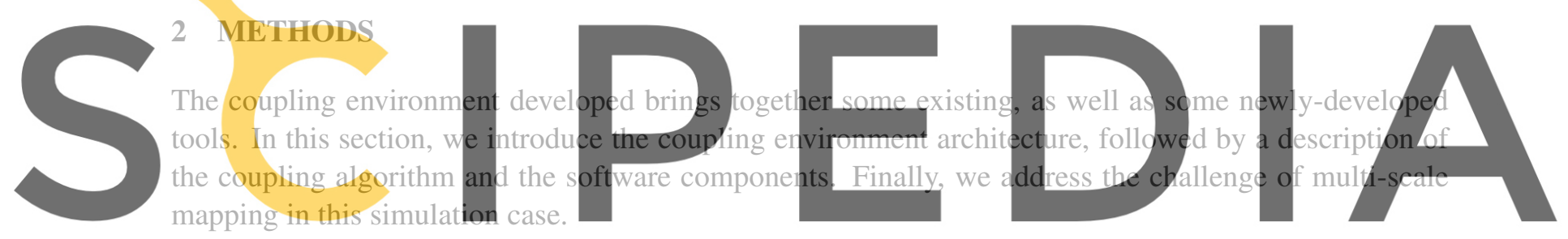

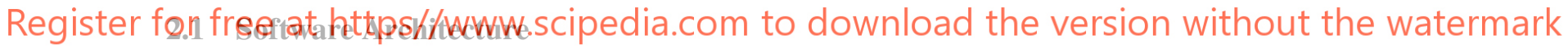

The main goals of this work include the modularity, extensibility, and ease-of-use of the developed coupling environment and the respective adapters. The main architectural challenges were the closedsource nature of the solvers and the lack of full-featured APIs or plugin interfaces. The interaction between the different software components is shown in Fig. 1. The TAU adapter ${ }^{3}$ is a Python script that combines calls to the preCICE library and the TAU-Python Application Programming Interface (API). The CAMRAD II adapter ${ }^{4}$ is a Python script that runs the CAMRAD II executable via bash. The two adapters modify the input files for the two solvers. Missing from this picture is the Python bindings of preCICE $^{5}$, which is assumed to be part of the preCICE component.

\footnotetext{
${ }^{1}$ preCICE website: https://www.precice.org/, source code: https://github.com/precice.

${ }^{2}$ preCICE community stories: https://www.precice.org/community-projects.html

${ }^{3}$ TAU adapter: https://gitlab.lrz.de/KeefeHuang/tauadapter, MIT license.

${ }^{4}$ CAMRAD II adapter: https://gitlab.1rz.de/KeefeHuang/camradadapter, MIT license.

${ }^{5}$ preCICE Python bindings: https://github.com/precice/python-bindings
} 


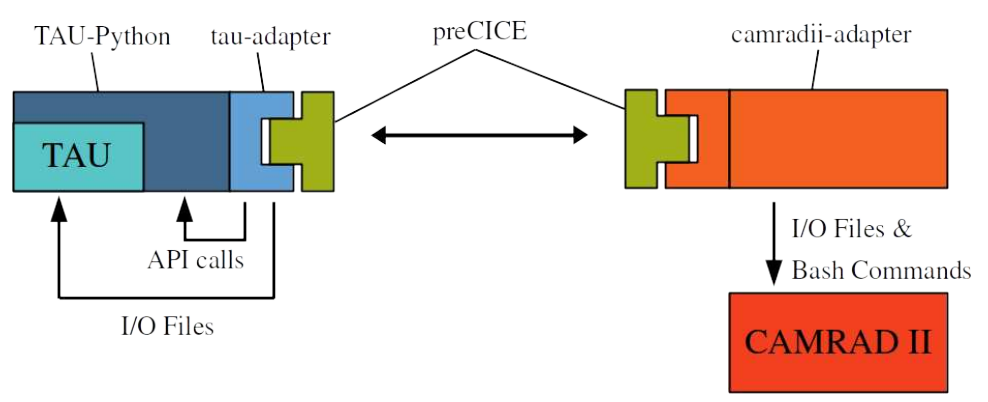

Figure 1: Overview of TAU and CAMRAD II Adapters.

\subsection{Coupling Workflow}

We use a coupling workflow similar to that employed by Potsdam in [4], also known as the delta airloads method; see Fig. 2. The CSD simulation starts and produces a trimmed solution: $F_{0}^{L L}$ and $M_{0}^{L L}$. The resulting blade motion is passed to the CFD simulation, which computes the aerodynamic loading data $F_{0}^{C F D}$ and $M_{0}^{C F D}$. The CSD simulation can then be restarted with corrections to the aerodynamic loading based on the CFD output. After the initial coupling step, a stopping condition is applied. Unlike the Potsdam example [4], which checks both input controls and aerodynamic loads, the simulation is assumed to have converged if the trimmed controls converge with a given absolute tolerance. In this simulation case,

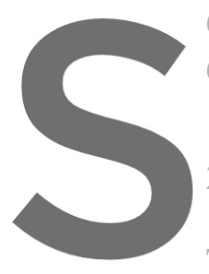
CAMRAD II and TAU CFD simulation is vastly

2.3 CAMRAD III Workflow

The first of the two solvers use
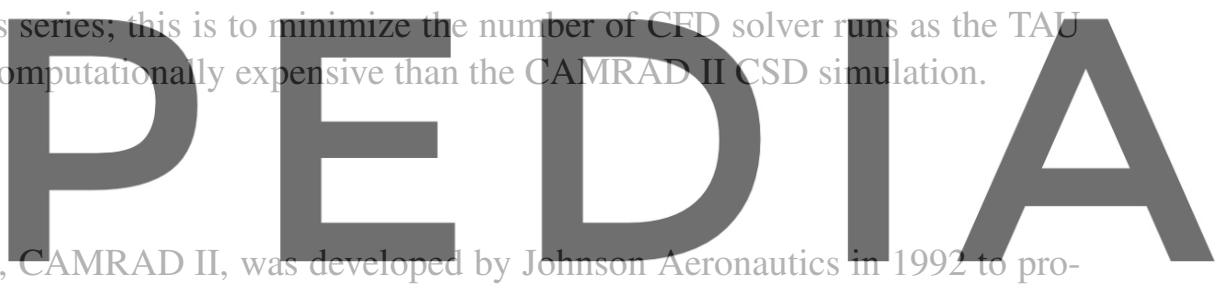

vide a rotorcraft comprehensive analysis tool; the software supports multi-body dynamics with a finite-

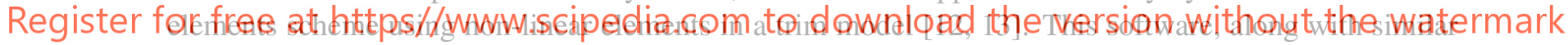

CSD codes, such as RCAS and DYMORE [14, 15], is one of the most widespread software solutions for structural dynamics codes in the field of rotorcraft technologies. CAMRAD II runs first in each iteration, sends updated motion and collocation point data to the TAU adapter, and receives aerodynamic loading and azimuth data at the start of each coupling iteration.

\subsection{TAU Workflow}

TAU is an finite volume method based CFD tool developed by the German Aerospace Center (DLR) to solve fluid flow problems around complex geometries, ranging from the subsonic to hypersonic regimes $[16,17,18]$. In this work, we used TAU v2018.1.0. In each coupling iteration, TAU receives motion data from CAMRAD II, which is used to update the blade motion in the CFD simulation. The TAU simulation is run for a user-defined number of blade revolutions, chosen such that a steady-state solution is reached before aerodynamic loading data is sent back to CAMRAD II. Before the first coupling with CAMRAD II, the TAU simulation is run for a user-defined number of initial revolutions. This removes the starting vortices around the rotor blades, increasing the stability of the coupled simulation. 


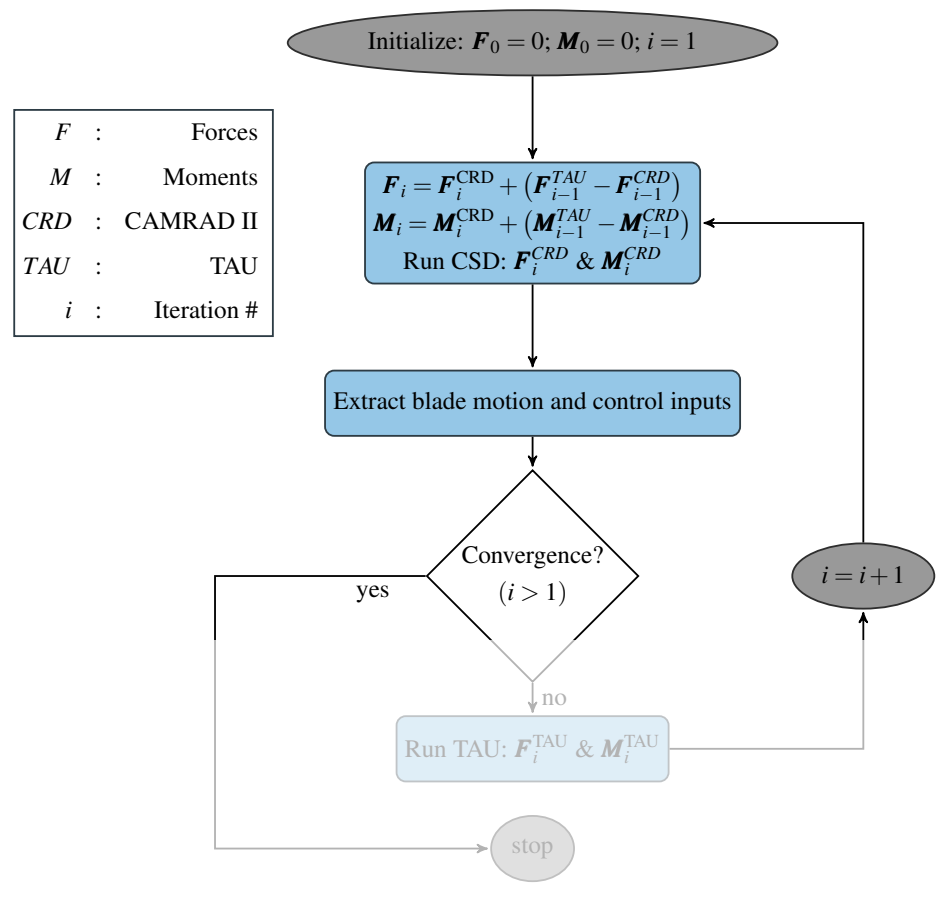

Figure 2: Modified Delta Airloads Algorithm Workflow [4].

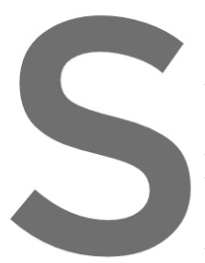

2.5 preCICE

preCICE ${ }^{6}$ is a free/open-source

Stuttgart [1]. The library

physics solvers using a partition
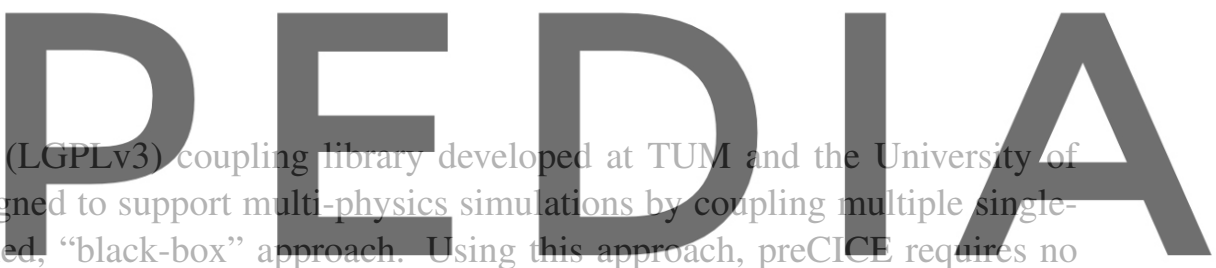

information about the inner workings of either solver. Instead, preCICE exchanges information at user-

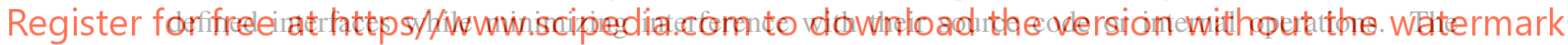
preCICE adapters, "glue codes" between the solvers and preCICE, extract and set data from/into the solvers' data structures, set the time step, and store checkpoints of the state of the solvers in case of an implicit (iterative) coupling scheme.

\subsection{Geometric Multi-Scale Mapping}

Mapping the aerodynamic load data from the 3D cells of TAU to the 1D panels of CAMRAD II requires special treatment due to its geometric multi-scale nature. While preCICE supports nearest-neighbor and nearest-projection data mapping, as well as Radial Basis Function (RBF) interpolation [1], none of them can be directly applied to this 3D-1D mapping, as they do not consider the panel edge positions where the properties of the blade can change sharply. This is a limitation of the current mapping implementations in preCICE, but such conversions can be handled in the adapters. Figure 3a shows a top-down view of a TAU 3D discretization (above) and a 1D CAMRAD II discretization below. The data values of each cell in the TAU discretization are mapped to the nearest collocation point (centroid of each panel) of the

${ }^{6}$ preCICE website: https://www.precice.org/, code: https://github.com/precice/precice 
same color below. Ideally, the red and blue divisions in the TAU discretization should match with the panel edges seen in the CAMRAD II discretization. However, this is not the case in Fig. 3a; the blue cells highlighted in orange are erroneously assigned to the first panel instead of the second panel as they are physically closer to the collocation point of the first panel.

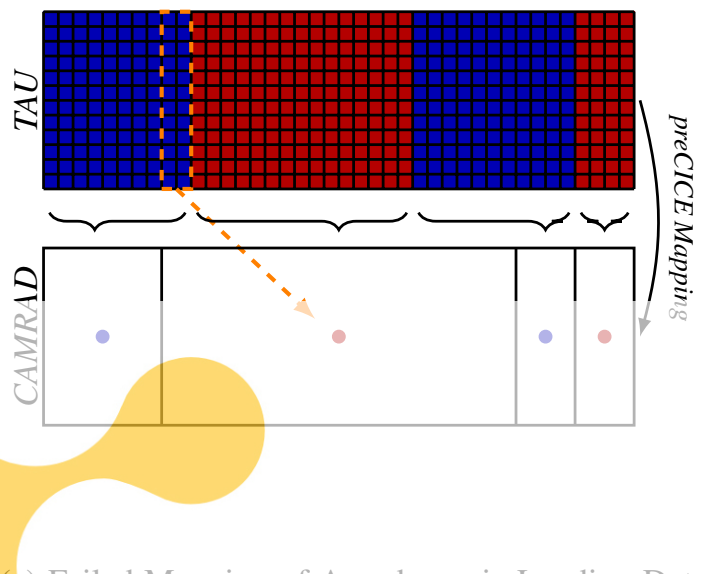

(a) Failed Mapping of Aerodynamic Loading Data from TAU (above) to CAMRAD II (below). Incorrectly mapped cells are highlighted in orange
Figure 3: Acrodynamic Load Mappi
To resolve this issue, we introduce a 2D intern

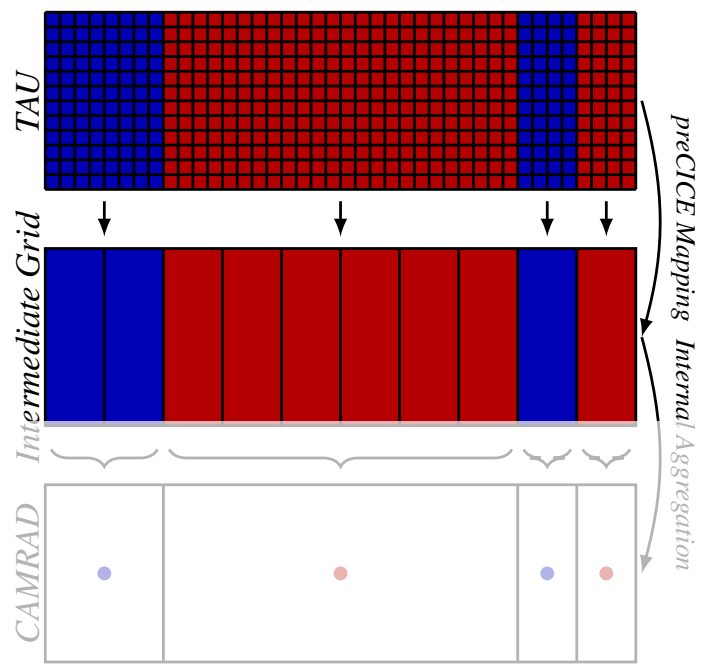

(b) Successful Mapping of Aerodynamic Loading Data from (above) to CAMRAD II (below) with In-

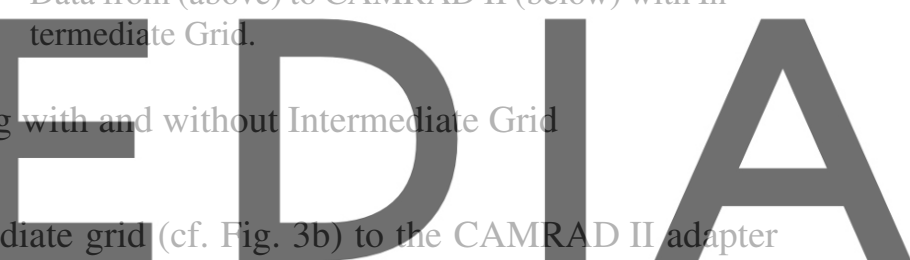

code, based on the CAMRAD II discretization. An additional internal aggregation step is then performed

Register for free at hAt II code to sum up the aerodynamic loadf at gach collocation point Before running simulations, the veracity of the new method was checked: We passed output forces from a prior test run between TAU and CAMRAD II via the intermediate grid, compared the values at each collocation point, and saw almost exact agreement; the resulting comparison can be seen in [19].

\section{SIMULATION CASE}

This section details the individual simulation setups of the two solvers. We use a rotor model based on the rotor tested in the second Higher-Harmonic Control Aeroacoustics Rotor Test (HART) [20]. For this purpose, a scaled four bladed Bo-105 main rotor for the TAU and CAMRAD II models was developed $[20,11]$. The simulation inputs were set to the baseline test conditions in the HART II test [21, 19]. One important modification to these parameters was to pitch the rotor pitch angle towards the front by $4.5^{\circ}$ instead of the rear [11]. The forward tilt of the rotor would reduces vortices on the blade surface, accelerating the convergence of the simulation. This is a simplified fluid model, intended to function as a proof-of-concept. A non-optimized mesh was used and only rigid body motion was considered. 


\subsection{CAMRAD II Simulation Setup}

The rotor blade used in this CAMRAD II model was divided into 10 sections (used for structural definition) and 21 collocation points (centroids where aerodynamic loads are applied). These can be seen in Fig. 4 and Fig. 5, respectively. Following the simulation logic, CAMRAD II runs iteratively to provide a comprehensive analysis of the helicopter rotor for given trim conditions. In this study, the rolling moment $(-20 \mathrm{Nm})$, pitching moment $(-20 \mathrm{Nm})$ and thrust $(3300 \mathrm{~N})$ were trim conditions.

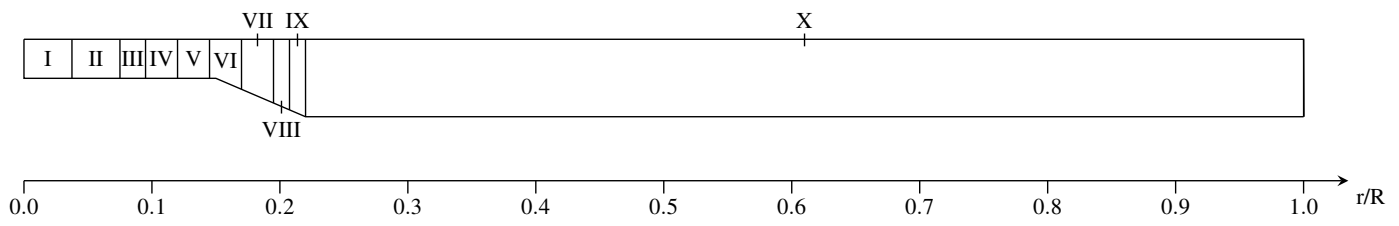

Figure 4: Locations of Sections defining the Structural Properties of Rotor Blades in CAMRAD II [11].

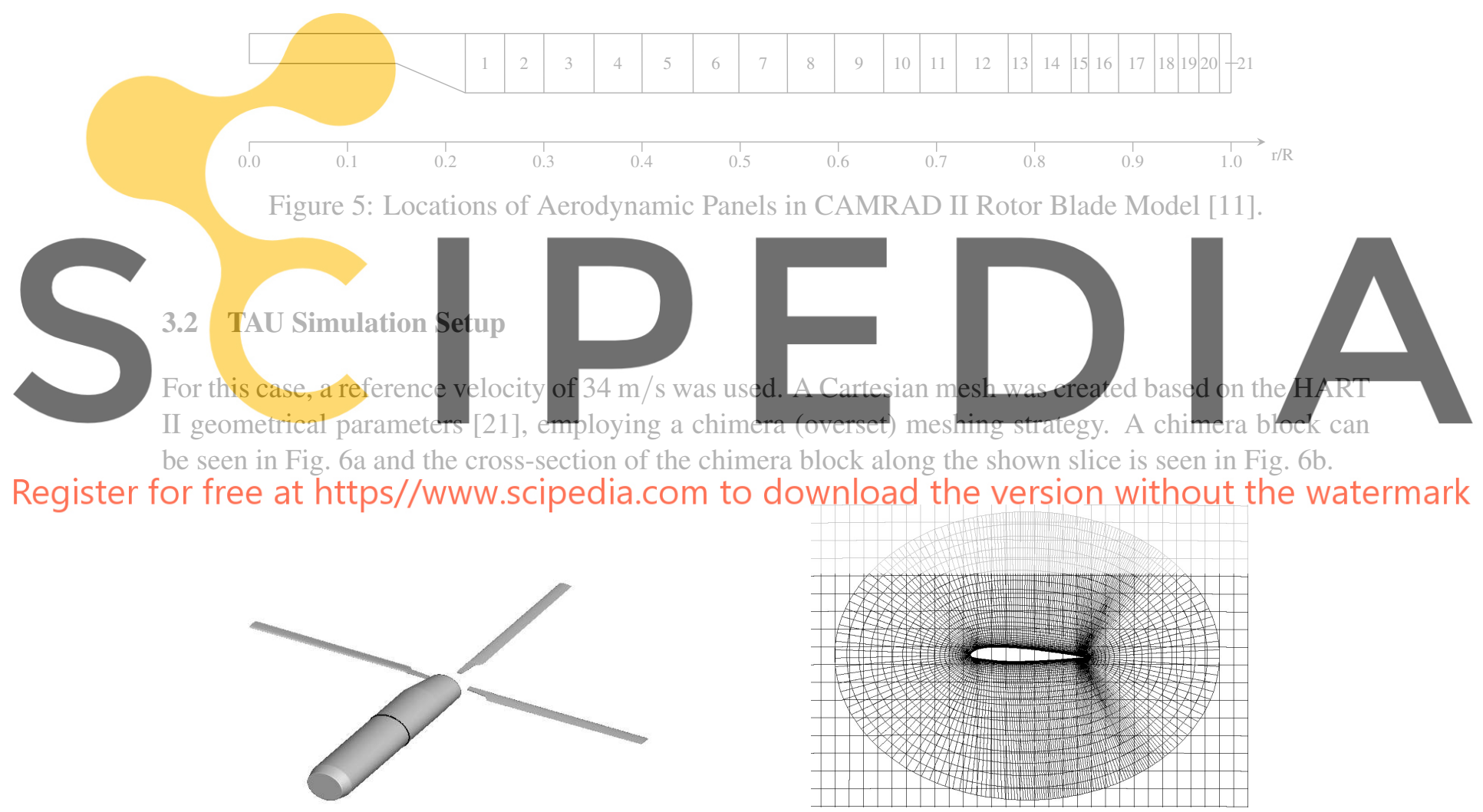

(a) Rotor Blade model with a highlighted single (b) Cross-section of Chimera block shown in (a). Chimera block.

Figure 6: Rotor Blade model for TAU.

A single blade was discretized and rotated multiple times to represent the four bladed rotor model. The TAU simulation used the unsteady Reynolds-averaged NavierStokes (URANS) equations with the 
one-equation Negative Spalart - Allmaras turbulence model [22], used for turbulence closure to ensure more solver robustness in comparison to the SA-Standard model. The three dimensional CFD grid had 22 Million cells. Moreover, a three-stage multi-grid method was applied at every time step to accelerate convergence. The CFL number used on the fine grid level was 6 . The convective RANS flux discretization was solved using the central scheme and the implicit backward Euler scheme was used for the time discretization. In order to achieve to achieve convergence, 360 time steps per revolution were used (one degree of rotor rotation per time step). A total of seven full initial revolutions were run in TAU, with 250 inner iterations per time step, prior to coupling. This was reduced to 100 inner iterations during the coupling to speed up the time to solution.

\section{RESULTS}

\subsection{Loose Coupling for Rigid Blade}

Seven coupling iterations were considered to evaluate the results of the CFD/CSD coupling, plotting the difference between the aerodynamic forces $\left(F_{x}, F_{y}, F_{z}\right)$ and moments $\left(M_{x}, M_{y}, M_{z}\right)$. We used the difference between the control angles extracted from the trimmed CAMRAD II solutions, pitch $(\Delta \theta)$, lead-lag $(\Delta \psi)$ and flap $(\Delta \phi)$, as a stopping criterion, see Fig. 7. The first data points of the figure correspond to the control angles prior to coupling. We set the stopping criterion to when $\Delta \theta, \Delta \psi$, and $\Delta \phi$ are less than or equal to $0.02 \mathrm{deg}$. For the first three coupling steps, the difference between the control angles changes remarkably. This indicates that the three-dimensional CFD aerodynamic forces and moments, given as

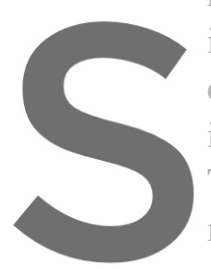
input to the CAMRAD I simulation, vary coupling steps, CAMRAD ison between the TAU anc

TAU output was obtained means of the lifting line the
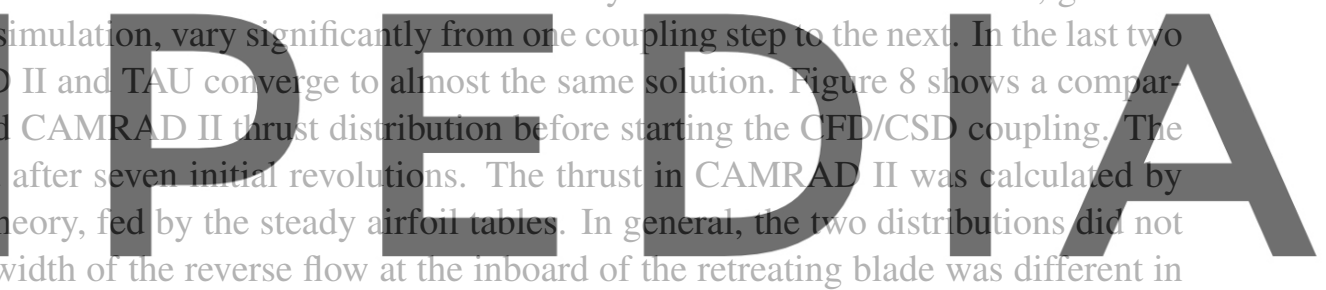

match. For example, the width of the reverse flow at the inboard of the retreating blade was different in each domain. The TAU simulation computed higher thrust values over the outboard side of the retreading

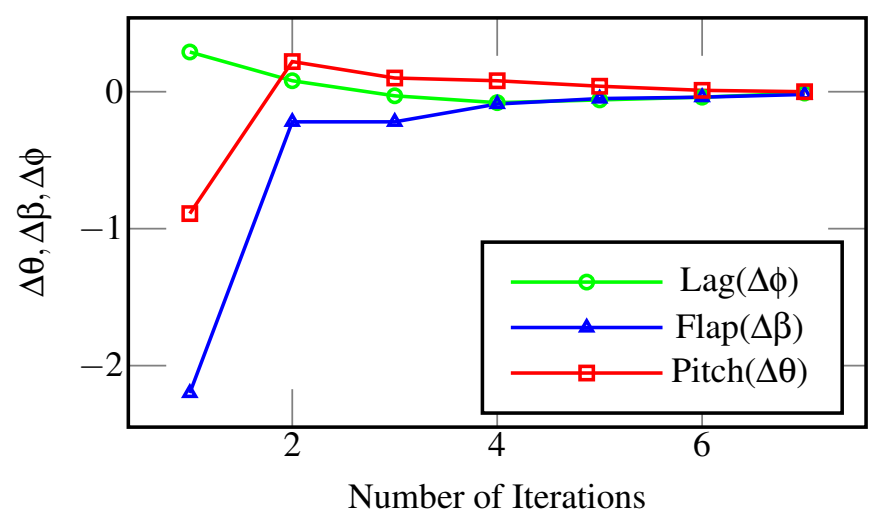

Figure 7: CAMRAD II control values per iteration number. 


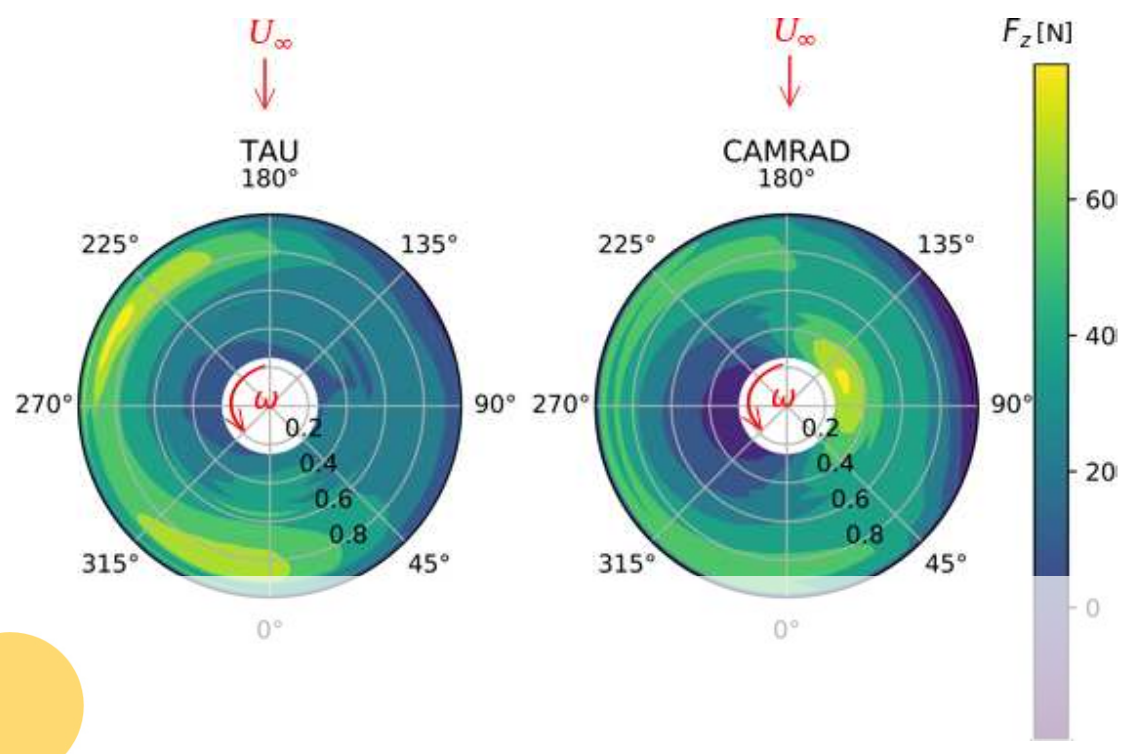

Figure 8: Comparison of TAU (left) and CAMRAD II (right) thrust distribution $\left(F_{z}\right)$ over a single revolution prior to coupling.
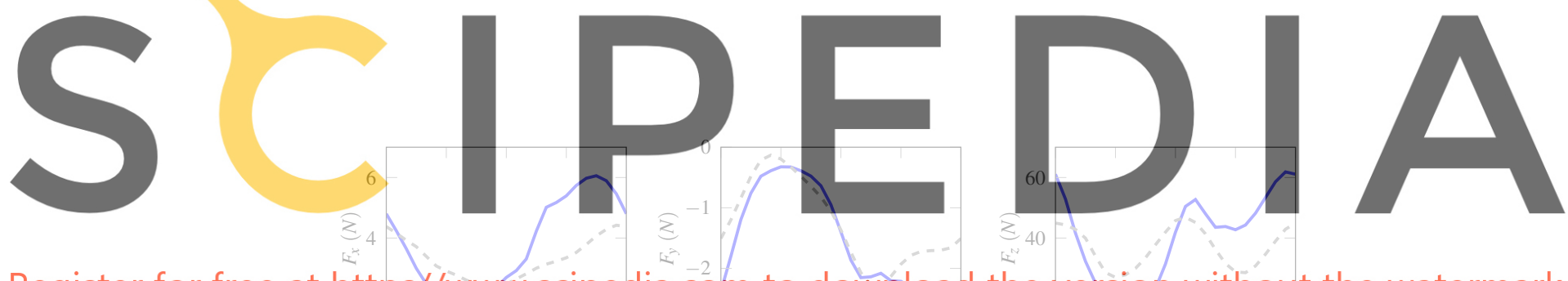

Register for free at https/Xwwrw.scipedia.com to downtoad the veksion without the watermark
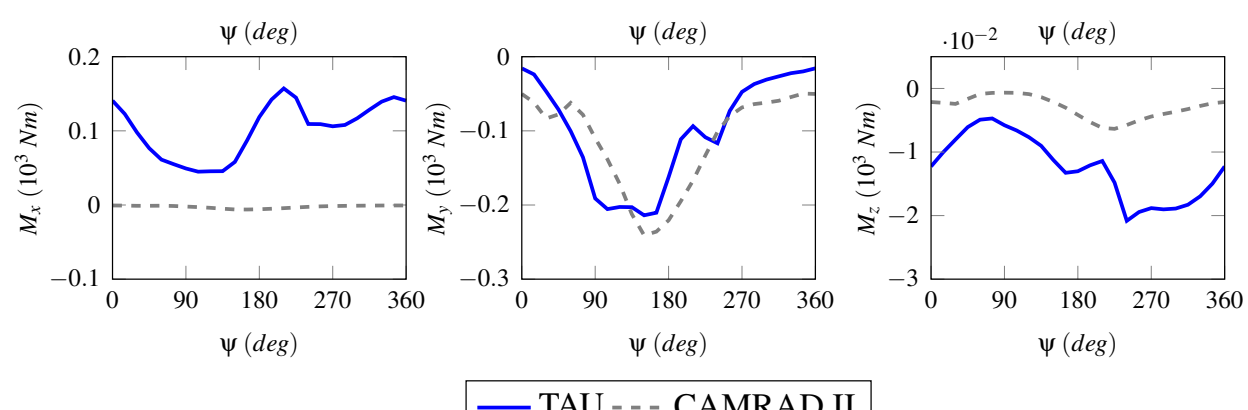

Figure 9: Comparison of TAU and CAMRAD II aerodynamic force and moment data over a single revolution prior to coupling at CAMRAD II sensor position $r=0.87$. 
Figure 10 shows how the thrust distributions varied after seven coupling iterations. Here, both solvers approximated the same width of the reverse flow region. Furthermore, the estimated thrust values over the retreating side was almost identical in comparison to the distribution obtained after three coupling steps. Slight deviations were observed at the advancing side, since the control angles used in TAU and CAMRAD II were not identical. The variations in aerodynamic forces $\left(F_{x}, F_{y}, F_{z}\right)$ and moments $\left(M_{x}, M_{y}, M_{z}\right)$ before coupling TAU and CAMRAD II, and after the third and the seventh coupling iteration are observed in order to evaluate the progress of the CFD/CSD coupling. The forces and moments were extracted at the $r / R=0.76$ CAMRAD II sensor position. Before starting the coupling (Figure 9), the three dimensional forces and moments of TAU differed significantly from the CAMRAD II results, computed by means of the lifting line theory. The differences in $M_{x}$ and $M_{z}$ over the azimuth angle $\psi$ were particularly strong. The forces $F_{x}, F_{y}$ and $F_{z}$ calculated by TAU and CAMRAD II had the same mean values over the azimuth angle $\psi$.

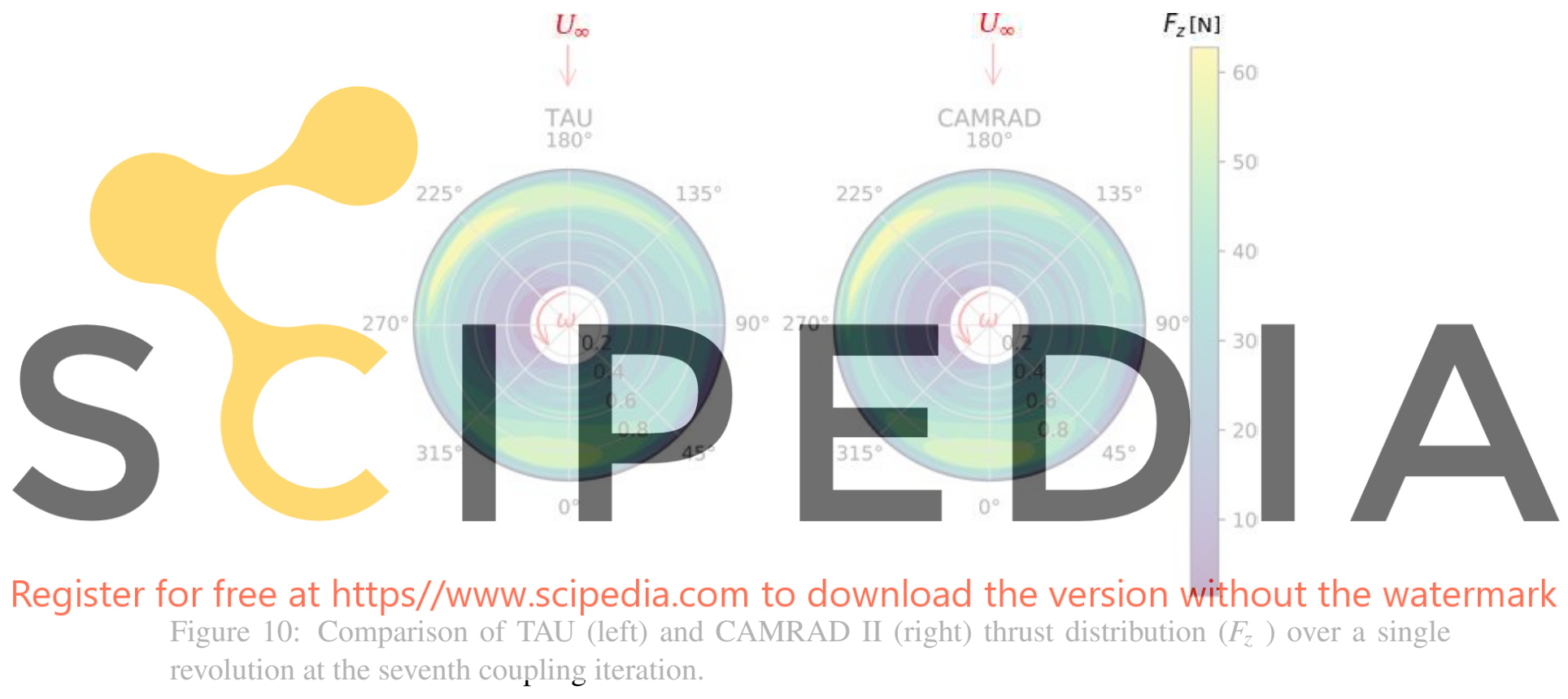

\subsection{Runtime Comparisons}

An important question and motivation behind this study was not only ensuring the accuracy, but also maintaining or even improving the runtime of the coupled simulation. We compare here the runtime of the TAU-preCICE adapter with the original implementation by Carnefix [11]. We focus on TAU, as the CAMRAD II runtime is negligible. Both setups were tested on the compute cluster of the Institute of Helicopter Technology, on a single node (64 cores, AMD-6376). TAU was executed on 24 processes and CAMRAD II serially. The two simulations were run with identical preprocessing, with zero inner iterations, and for two full coupling iterations. In Fig. 12, we present a comparison of the cumulative setup time and coupling time per iteration. We focus only on these phases, as the rest of the simulation runtime remains the same. The setup time for the in-house coupling is not available, as the coupling and 

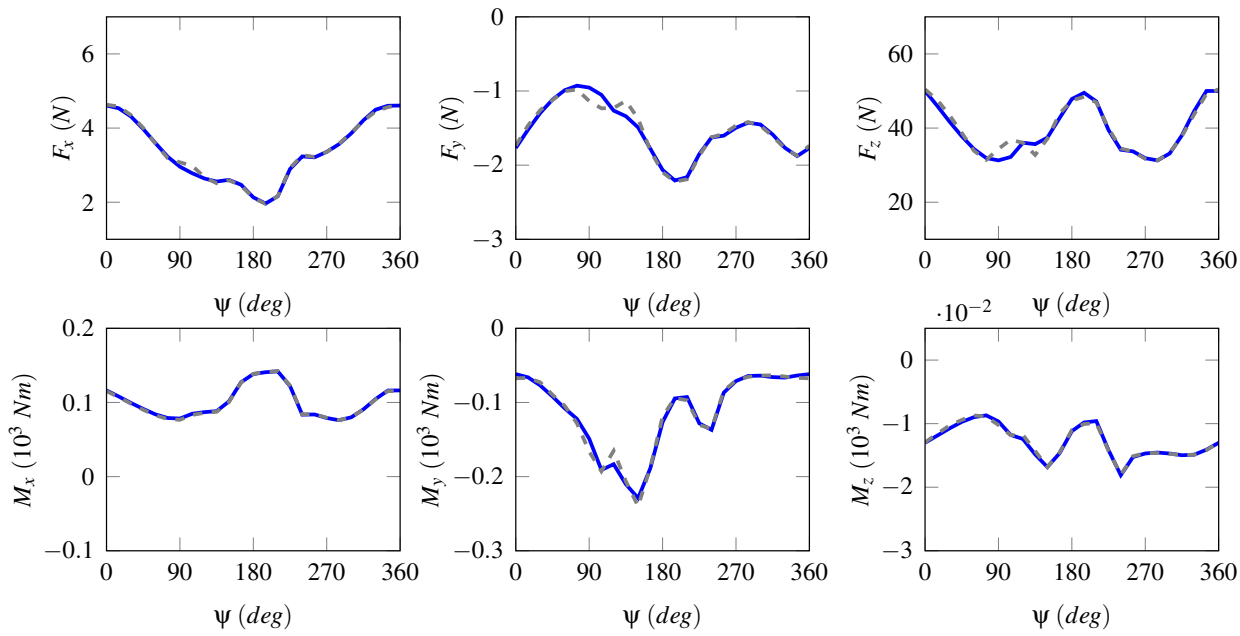

— TAU $=--$ CAMRAD II

Figure 11: Comparison of TAU and CAMRAD II aerodynamic force and moment data over a single revolution after the seventh coupling iterations at CAMRAD II sensor position $r / R=0.76$.

simulation setup phases were intertwined. Even when assuming no setup time for the original implementation, the preCICE-based coupling has significantly lower total time, as the adapter code runs faster within one coupling iteration by roughly a factor of 33. The main reason for this runtime decrease was parallel processing inside the context of the complete simulation, as the coupl can clearly show that intr that it better prepares the coupling environment fo
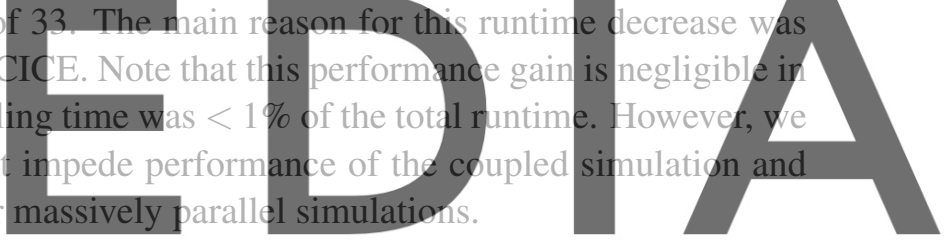

Register for free at https//www.scipedia.com to download the version without the watermark In-house Coupling

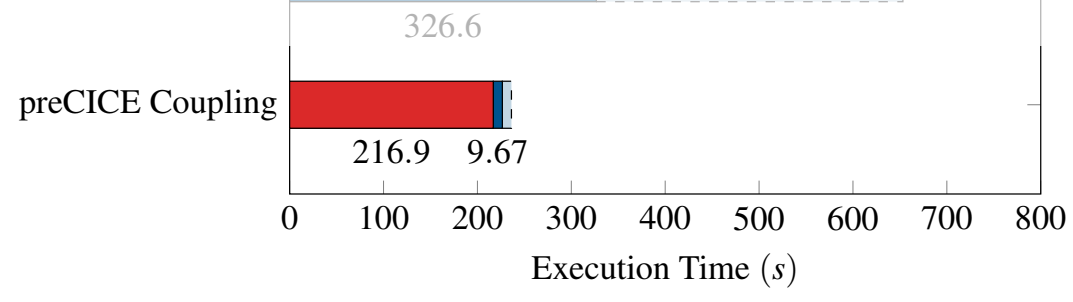

$\square$ Setup Time $\square$ Coupling Iteration 1

Figure 12: Bar graph showing setup time and coupling time. 


\section{CONCLUSION}

We presented a modular, extensible, and easy-to-use coupling environment for rotorcraft FSI simulations, which agrees numerically with our previous in-house coupling implementation, allows for further parallelization, and improves the coupling runtime by a factor of 33 per iteration in the studied scenario of a modified HART II rotor case. We used preCICE in the core of our coupling environment, overcoming a set of challenges uncommon to preCICE-based simulations in the literature. First, we interacted with the closed-source solvers TAU and CAMRAD II using the TAU-Python API and I/O files for the former, and a run script, and I/O files for the latter, a decision that made the coupling possible but leaves potential for further performance improvements. Second, we coupled periodic data using a different set of preCICE data for each sampled azimuth, a successful workaround which calls for new configuration options in preCICE. Third, we coupled the 1D CAMRAD II domain with the 3D TAU domain using an intermediate grid to transform the data in the CAMRAD II adapter. This intermediate grid also served as a mechanism to map the aerodynamic loads to the correct panels of the non-homogeneous blade. However, this intermediate mesh needs to be aligned with the blade panels, a requirement that might be too restrictive in the future. This work is just a first step in a larger project to simulate a rotor blade camber morphing using such a coupled simulation. This will require the TAU adapter to handle cases with a larger number of controlled deformations (induced by the FishBAC mechanism). The coupling environment that we created provides a solid foundation for this goal, and the flexibility of preCICE will facilitate further investigations with different models, algorithms, and solvers.

\section{ACKNOWLEDGEMENTS}

This study has conducted within the scope of the SABRE project. This project has received funding from the European Union's Horizon 2020 research and innovation program under grant agreement No. 723491. We would like to thank Stefan Platzer for his contributions and feedback. Benjamin Uekermann has been funded by the European Union's Horizon 2020 research and innovation program under the Marie Sklodowska-Curie grant agreement No 754462 and by the DFG Cluster of Excellence EXC 2075 Data-Integrated Simulation Science (SimTech). Gerasimos Chourdakis has been funded by the German Federal Ministry for Economic Affairs and Energy (BMWi) project number 1501593.

\section{REFERENCES}

[1] H.-J. Bungartz, F. Lindner, B. Gatzhammer, M. Mehl, K. Scheufele, A. Shukaev, and B. Uekermann, "preCICE - a fully parallel library for multi-physics surface coupling," Computers and Fluids, vol. 141, pp. 250-258, 2016. Advances in Fluid-Structure Interaction.

[2] K. Pahlke and B. van der Wall, "Calculation of Multibladed Rotors in High-Speed Forward Flight with Weak Fluid-Structure-Coupling," in 27th European Rotorcraft Forum, 2001.

[3] G. Servera, P. Beaumier, and M. Costes, "A weak coupling method between the dynamics code HOST and the 3D unsteady Euler code WAVES," Aerospace Science and Technology - AEROSP SCI TECHNOL, vol. 5, pp. 397-408, 2001.

[4] M. Potsdam, H. Yeo, and W. Johnson, "Rotor airloads prediction using loose aerodynamic/structural coupling," Journal of Aircraft, vol. 43, no. 3, pp. 732-742, 2006. 
[5] W. Johnson, "Technology Drivers in the Development of CAMRAD II," 1999.

[6] A. Altmikus, S. Wagner, P. Beaumier, and G. Servera, "A comparison: Weak versus strong modular coupling for trimmed aeroelastic rotor simulations," in American Helicopter Society 58th Annual Forum, 2002.

[7] J. Abras, C. Lynch, and M. Smith, "Advances in Rotorcraft Simulations with Unstructured CFD," Annual Forum Proceedings - AHS International, vol. 3, 2007.

[8] R. Marpu, L. Sankar, T. Norman, T. Egolf, and S. Makinen, "Analysis of the UH-60A rotor loads using wind tunnel data," in 51st AIAA Aerospace Sciences Meeting including the New Horizons Forum and Aerospace Exposition 2013, 2013.

[9] R. Biedron and E. Lee-Rausch, "Computation of uh-60a airloads using CFD/CSD coupling on unstructured meshes," American Helicopter Society 67th Annual Forum, 2011.

[10] D. Schwamborn, A. Gardner, H. von Geyr, A. Krumbein, H. Lüdeke, and A. Stürmer, "Development of the TAU-code for aerospace applications," 2008.

[11] A. Carnefix, "Development of a framework for trimmed rotorcraft simulations using loose coupling of cfd and csd software," Master's thesis, Technical University of Munich, 2017.

[12] L. Ahaus, S. Makinen, T. Meadowcroft, H. Tadghighi, L. Sankar, and J. Baeder, "Assessment of CFD/CSD analytical tools for improved rotor loads," Annual Forum Proceedings - AHS International, vol. 2, pp. 1164-1186, 2015.

[13] W. Johnson, "A history of rotorcraft comprehensive analyses," NASA TP 2012-216012, 2012.

[14] H. Saberi, M. Khoshlahjeh, R. Ormiston, and M. Rutkowski, "Overview of rcas and application to advanced rotorcraft problems," AHS International 4th Decennial Specialists' Conference on Aeromechanics, pp. 741-781, 2004.

[15] O. Bauchau, "Computational schemes for flexible, nonlinear multi-body systems," Multibody System Dynamics - MULTIBODY SYST DYN, vol. 2, pp. 169-225, 1998.

[16] D. Schwamborn, T. Gerhold, and R. Heinrich, "The DLR TAU-code: Recent applications in research and industry," 2006.

[17] T. Gerhold, M. Galle, O. Friedrich, and J. Evans, "Calculation of complex three-dimensional configurations employing the DLR-TAU-code," in 35th Aerospace Sciences Meeting and Exhibit, AIAA ARC, 1997.

[18] D. S. T. Gerhold, V. Hannemann, "On the validation of the DLR-TAU code," in New Results in Numerical and Experimental Fluid Mechanics II - Contributions to the 11th AG STAB/DGLR Symposium, Berlin, Germany, 1998 (W. Nitsche, H.-J. Heinemann, and R. Hilbig, eds.), pp. 426-433, Berlin, Heidelberg: Springer, 1999.

[19] Q. Huang, "Loose coupling of isolated rotorblade rotorcraft CFD/CSD simulations using preCICE," Master Thesis, Technical University of Munich, 2019.

[20] B. Wall, "2nd HHC aeroacoustic rotor test (HART II) - Part I: Test documentation -," 2003.

[21] M. Smith, J. Lim, B. Wall, J. Baeder, R. Biedron, D. Jr, B. Jayaraman, S. Jung, and B.-Y. Min, "The HART II international workshop: an assessment of the state of the art in CFD/CSD prediction," CEAS Aeronautical Journal, vol. 4, 2013.

[22] S. R. Allmaras, F. T. Johnson, and P. R. Spalart, "Modifications and clarifications for the implementation of the spalart-allmaras turbulence model," in 7th Int. Conf. on Comp. Fluid Dyn., 2012. 